\title{
Método para la selección de casos de estudio: adaptación climática del Movimiento Moderno en el clima cálido ecuatorial
}

\author{
Iván Osuna-Motta ${ }^{1}$ | Paulina Wegertseder-Martinez ${ }^{2}$ | Carolina M. Rodríguez ${ }^{3}$
}

Recibido: 29-03-2021| Versión final: 24-06-2021

Resumen

\begin{abstract}
Las normas que obligan a la reducción en el consumo energético en las edificaciones son cada vez más estrictas, y tienen como meta a mediano plazo, lograr edificios con consumo cero. La necesaria actualización de los edificios patrimoniales enfrenta varias limitaciones, ya que las normas de protección reducen las posibles intervenciones, conducentes a la eficiencia energética y el confort de los usuarios. Este artículo presenta una metodología replicable que identifica las tipologías de envolvente profunda más utilizadas durante los inicios del Movimiento Moderno en Cali, Las clasifica según sus características geométricas y determina las más representativas. El objetivo del artículo es caracterizar las tipologías existentes y generar una clasificación que pueda ser empleada en el futuro para determinar su eficiencia respecto a la calidad del ambiente interior en el clima cálido ecuatorial. Para definir la muestra intencional no probabilística, se determinó un universo amplio y se fue depurando con criterios de usos, alturas, año de realización y estado de conservación de los edificios. La definición de unos criterios específicos permitió identificar las tipologías de envolvente profunda utilizadas en los edificios de oficinas construidos en Cali en los años 1950s, se clasificaron en 3 grupos: con elementos verticales, con elementos horizontales y con combinación de elementos verticales y horizontales. Finalmente se seleccionaron los edificios que mejor representan las envolventes profundas en Cali y que servirán como casos de estudio para identificar estrategias replicables en el diseño de fachadas.
\end{abstract}

Palabras clave: Patrimonio moderno construido; dispositivos de sombreado; bioclimática ecuatorial; diseño de fachadas

Citación

Osuna-Motta, et al. (2021). Método para la selección de casos de estudio: adaptación climática del Movimiento Moderno en el clima cálido ecuatorial. ACE: Architecture, City and Environment, 16(47) 10452. DOI: http://dx.doi.org/10.5821/ace.16.47.10452

\section{Case Studies Selection Method: Climate Adaptation of the Modern Movement in the Equatorial Warm Climate}

Abstract The regulations that aim to reduce energy consumption in buildings are increasingly strict, and their medium-term goal is to achieve buildings with zero consumption. The necessary updating of heritage buildings faces several limitations, since protection standards reduce possible interventions, leading to energy efficiency and user comfort. This article presents a replicable methodology that identifies the most used deep-envelope typologies during the beginnings of the Modern Movement in Cali, Colombia. It classifies them according to their geometric characteristics, highlighting the most representative ones. The article's objective is to analyse existing typologies, creating a classification that can be used to determine their efficiency regarding indoor environment quality in hot equatorial climates. A wide review of data was used to define a purposive non-probabilistic sample, refined according to building use, height, year of construction, and conservation condition. These criteria allowed to identify the types of deep-envelope used in the office buildings built in Cali in the 1950s: with vertical elements, with horizontal elements, and with a combination of vertical and horizonta elements. Finally, the buildings that best represented the deep-envelope systems in Cali were selected and used as case studies to identify replicable strategies for the design of facades.

Keywords: Modern built heritage; shading devices; equatorial bioclimatic architecture; façade design

${ }^{1}$ Magister en Arquitectura y Urbanismo, Profesor de planta de la Pontificia Universidad Javeriana de Cali, Doctorante en Arquitectura y Urbanismo de la Universidad del Bío-Bío (ORCiD: 0000-0001-5137-2603; Wos Researcher $\mathrm{ABH}-8430-2020),{ }^{2}$ Dra. Arquitecta, Profesora de la Universidad del Bío-Bío (ORCiD: 00000002-3485-9839), ${ }^{3}$ Dra. Arquitecta, Profesora del programa de Arquitectura de la Universidad Piloto de Colombia (ORCiD: 0000-0003-3409-7886) Correo de contacto: ivan.osuna@javerianacali.edu.co 


\section{Introducción}

En este artículo se presenta una propuesta metodológica replicable por otros investigadores para determinar una muestra deliberada no probabilística con la cual se seleccionaron los casos de estudio del proyecto de tesis doctoral del autor principal. El muestreo intencional o deliberado es una técnica en la cual el investigador, con base en su juicio y conocimiento, selecciona los individuos que harán parte de la muestra, y que permiten satisfacer las necesidades específicas del proyecto.

La eficiencia energética y el confort térmico de los edificios históricos se ha convertido en los últimos años en un tema de gran interés ya que se ha demostrado que adaptar el patrimonio arquitectónico a los nuevos estándares de confort y eficiencia energética es necesario para lograr la sostenibilidad y la conservación de los edificios históricos (Lidelöw et al., 2019). La mayor parte de los estudios encontrados se centran en el patrimonio construido en el siglo XX, que en muchos casos conservan su uso original y por lo tanto, su adecuación genera un impacto inmediato en el ahorro energético y el confort de sus usuarios (Martínez-Molina et al, 2016). Adicionalmente se ha demostrado que, en muchos casos, los edificios patrimoniales poseen un gran potencial de ahorro energético, ya que su adaptación al clima se ha hecho con criterios de diseño arquitectónico, en épocas en las cuales no existían grandes desarrollos tecnológicos para la climatización (Sugár et al., 2020).

Evitar el sobrecalentamiento de los edificios en los climas cálidos representa uno de los actuales desafíos de la sostenibilidad debido a su alto impacto en la reducción en el consumo energético, para lo cual se requieren criterios claros que consideren las condiciones ambientales y los aspectos culturales del lugar específico en que se diseña un edificio (Triana et al., 2020). El uso generalizado de fachadas vidriadas o con dispositivos externos "de catálogo" que atienden criterios de mercadeo, se ha convertido en un nuevo estilo internacional, que en muchos casos no considera las condiciones climáticas específicas de cada lugar, y como consecuencia se genera un alto consumo energético durante toda la vida útil de los edificios (Keitsch, 2012; Patsavos \& Zavoleas, 2014). Una manera de hacer frente a esta problemática consiste en entender cómo algunos edificios han resuelto de manera exitosa su relación con el clima, sin la necesidad de utilizar medios mecánicos de climatización que consumen una gran cantidad de energía. Con ese criterio, se plantea la necesidad de estudiar los diseños de las fachadas implementadas por el Movimiento Moderno en los climas tropicales, las cuales pueden actuar como un sistema de intermediación entre los espacios interiores y exteriores, sin requerir de un elevado consumo energético para lograr unos niveles adecuados de confort térmico, acústico y lumínico (Ali, 2000; Requena-Ruiz, 2016; Requena Ruiz, 2011).

Durante los últimos años ha aumentado de manera significativa el número de trabajos de investigación acerca de los dispositivos de sombreado, especialmente para edificios de oficinas, lo cual ha demostrado que el diseño de la envolvente afecta directamente el rendimiento térmico y el consumo de energía (Freewan, 2014; Kirimtat et al., 2016; Mirrahimi et al., 2016; Sadineni et al., 2011; U.S. Departament of Energy, 2012). Los elementos de sombreado externos en las fachadas han sido clasificados por varios autores, quienes coinciden en determinar tres tipologías básicas: horizontal, vertical y combinada, clasificadas según la dirección de los elementos que la conforman y los niveles de ocultación del cielo que proveen, tal y como se muestran en la Figura 1. La última de estas, también conocida como egg-crate, se caracteriza por la combinación de elementos horizontales y verticales, con composiciones de celosías, rejillas o marcos (Arifin \& Denan, 2015; Ghosh \& Neogi, 2018; Lau et al., 2016; Olgyay, 1968).

\footnotetext{
${ }^{1}$ El término Movimiento Moderno utilizado en este artículo se refiere al estilo arquitectónico desarrollado en todo el mundo occidental, especialmente entre 1925 y 1965. Su denominación cambia entre los países, siendo también llamado Racionalismo o Estilo Internacional.
}

ACE, 16 (4.7) CC BY-ND 3.0 ES | UPC Barcelona, España | Método para la selección de casos de estudio: adaptación 
Figura 1. Tipologías de dispositivos de sombreado (parasoles) y su correspondiente máscara de sombras (proyecciones)

HORIZONTAL
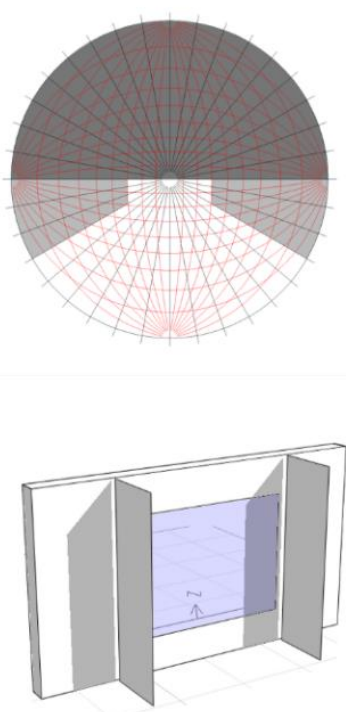

VERTICAL
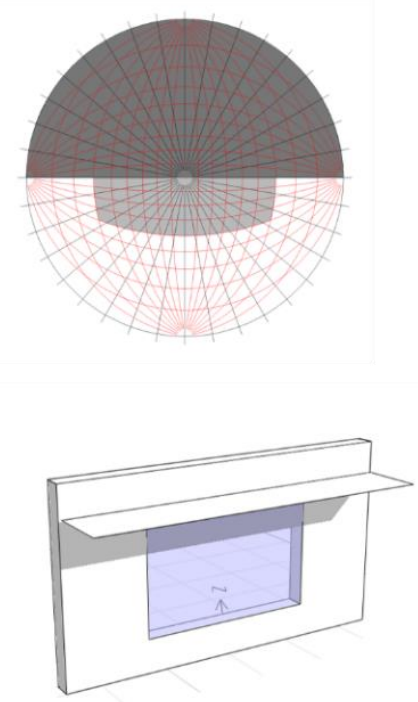

COMBINADO
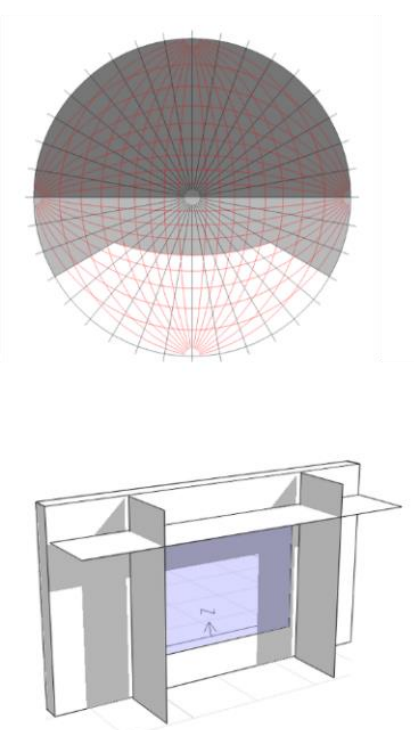

Fuente: Elaboración propia basada en Olgyay (1968)

El desempeño bioclimático de la Arquitectura Moderna ha sido estudiado por varios autores, centrándose en edificios paradigmáticos diseñados por importantes arquitectos como Le Corbusier - Lucio Costa para regiones subtropicales o templadas del planeta. En algunos casos se logró demostrar la eficacia de las estrategias de diseño pasivo implementadas, pero en otros casos se evidenció la poca efectividad de los parasoles o brise-soleils como dispositivo para proteger los espacios interiores de la radiación solar directa (Alvarenga, 2013; Melendo et al., 2008; Roesler et al., 2018; Siret et al., 2011). Para el caso específico de Colombia, aunque hay poca literatura al respecto, se ha demostrado que desde la década de 1940s, algunos jóvenes arquitectos comenzaron a implementar envolventes profundas, diseñadas a partir de un cuidadoso estudio de la geometría de la aparente trayectoria solar en la latitud específica de Cali, logrando una adecuada protección de la radiación solar directa, sin sacrificar los niveles de iluminación natural en los espacios interiores (Galindo-Díaz et al., 2020).

La arquitectura moderna en Latinoamérica fue muy valorada por instituciones políticas, culturales y comerciales que la convirtieron en un símbolo de progreso económico y tal vez por esto en Colombia muchos de los mejores edificios de este periodo se construyeron para sedes de entidades financieras, Las condiciones climáticas del trópico permitieron el desarrollo del concepto de brise-soleil que había sido propuesto por Le Corbusier pero que no había tenido buenos resultados en países con clima templado. Sin embargo, gran parte de esta arquitectura ha sido muy poco estudiada y se presenta una gran desproporción entre algunas figuras muy reconocidas y muchos arquitectos anónimos (Rovira y Gastón, 2007).

La arquitectura moderna colombiana ha sido escasamente difundida en el ámbito internacional y solo algunas investigaciones, como la realizada por Mayorga y Fontana (2006) han tratado de visibilizarla, resaltando su respuesta a criterios de eficiencia y modernidad, con propuestas 
universales sin dejar de expresar un auténtico carácter nacional. Una verdadera arquitectura del lugar caracterizada por el dominio del oficio. José María Sostres (1983) citado por Llanos (2008) asegura que: "La preponderancia del factor urbanístico y de las condiciones climatológicas condiciona el carácter de la arquitectura colombiana, que estilísticamente podemos considerarla como un estadio intermedio entre el racionalismo de Harvard y las sensacionales audacias del manierismo brasileño. Un tono comedido y realista domina esta variante de la arquitectura, en la que abundan aquellos elementos prácticamente justificados por los rigores de la temperatura, como patios interiores, brisesoleils y persianas, grandes voladizos protectores de la acción de los rayos solares, ingredientes que contribuyen a prestarle un genuino carácter nacional." (pág. 55)

Si bien esta afirmación está específicamente referida a la obra de la firma Obregón \& Valenzuela en la ciudad de Bogotá, Guzmán (2013) ha planteado la aplicabilidad de ese texto para la arquitectura construida en Cali por importantes firmas de arquitectos que han sido menos reconocidas a nivel nacional. La producción arquitectónica del Movimiento Moderno en Cali ha sido muy poco referenciada y las pocas publicaciones existentes se concentran en estudiar la obra de la firma Borrero, Zamorano \& Giovanelli (Tascón, 2000), aun cuando se ha identificado que en Cali, se desarrolló una arquitectura moderna propia, que daba una respuesta efectiva a condiciones ambientales del lugar, con una marcada influencia de la arquitectura carioca, debido a la similitud climática entre las dos regiones en términos de temperatura y brillo solar (Botti, 2019).

El concepto de envolvente profunda, que se introduce en esta investigación, ha sido referenciado en la literatura especializada con poca frecuencia. Referirse a la fachada de los edificios como envolvente es común, cuando se le asigna al cerramiento la responsabilidad de integrar de manera holística variables estéticas, climáticas, espaciales y culturales (Lovel, 2013). La referencia exacta al término aparece en el libro Deep Envelope, Thick Elevation, and All Things Architectural on the Equator de Erik L'Heureux (2016), fruto de su investigación en arquitectura para las ciudades de la zona ecuatorial. También se destaca el trabajo de Timo Carl (2019) en su libro Deep Skin Architecture, que investiga el potencial de las fachadas concebidas como pieles profundas para generar espacios intersticiales habitables, que realizan intermediación entre el interior y el exterior, y un control climático basado en el diseño.

En el contexto local, solo se encontró la investigación de Bustamante (2014) titulada La Profundidad de la Envolvente, que demuestra que la fachada arquitectónica durante el Movimiento Moderno aumento su espesor y adquirió una profundidad inédita, que alteró el concepto del límite entre el interior y exterior, dando a la envolvente la posibilidad de generar una experiencia sensorial y arquitectónica. En la presente investigación la envolvente profunda se define como el sistema de fachadas espaciales, utilizadas en los edificios del temprano movimiento moderno tropical, como una estrategia de intermediación, para lograr adecuados niveles de calidad del ambiente interior

En el Movimiento Moderno, la envolvente se libera de la estructura y adquiere un nuevo carácter espacial que le permite resolver desde el diseño, la intermediación con el ambiente exterior. Así, la fachada se convierte en el testigo del pensamiento moderno en el trópico, concepción que aún no ha sido estudiada con una aproximación holística que permita generar criterios de diseño replicables.

El objetivo de este artículo es presentar la metodología que permitió caracterizar las tipologías de los sistemas de envolvente profunda utilizados por el Movimiento Moderno en el clima cálido ecuatorial para controlar la radiación solar y regular los niveles de iluminación como primer paso para entender su impacto en la calidad del ambiente interior permitiendo así, identificar estrategias de diseño de fachadas como sistemas pasivos replicables para el desarrollo edificios de oficinas nuevos y futuros.

ACE, 16 (4.7) CC BY-ND 3.0 ES | UPC Barcelona, España | Método para la selección de casos de estudio: adaptación climática del Movimiento Moderno en el clima cálido ecuatorial. DOI: http://dx.doi.org/10.5821/ace.16.47.10452 


\section{Metodología}

El estudio se desarrolló en la ciudad de Cali en Colombia, localizada a 1.000 metros sobre el nivel del mar, en las coordenadas $3^{\circ} 27^{\prime} \mathrm{N}$ y $76^{\circ} 32^{\prime} \mathrm{O}$, con una temperatura promedio de $24^{\circ} \mathrm{C}$ y una humedad relativa de 75\% (CVC, DAGMA, \& CIAT, 2016). La ciudad posee un clima tropical de sabana semihúmedo (aw) de acuerdo con la clasificación de Köppen-Geiger, con una temperatura media de $24^{\circ} \mathrm{C}$ y una humedad relativa de 75\%, condiciones que tienen muy poca variación a lo largo del año. La variable climática más representativa es la oscilación diaria con $19^{\circ} \mathrm{C}$ y $95 \%$ de HR a las 6:00 de la mañana y hasta $31^{\circ} \mathrm{C}$ y $55 \%$ de $\mathrm{HR}$ a las 3:00 de la tarde. Debido a la baja latitud de la ciudad, la radiación solar incidente en las edificaciones se concentra principalmente sobre las cubiertas, seguido por las fachadas oeste y este. Sin embargo, las fachas sur y norte reciben al menos un $12 \%$ de la radiación solar durante el año (Osuna-Motta, 2017).

Se utiliza el estudio de casos, tal y como lo detalla Yin (2017), como una estrategia para hacer investigación empírica de un fenómeno particular dentro de su contexto real, utilizando múltiples fuentes de evidencia. El estudio emplea muestreo intencional que utiliza el juicio de los investigadores como criterio básico de selección, y construye una muestra que permite satisfacer sus necesidades específicas (Robson \& McCartan, 2016). En cualquier investigación, el rigor científico está determinado por la aplicación de un diseño de muestreo adecuado para el logro de los objetivos. En la investigación cuantitativa la selección de la muestra se realiza generalmente con criterios probabilísticos muy definidos en la literatura, pero no sucede de la misma manera en la investigación cualitativa, en la cual el investigador debe realizar una selección de un reducido número de casos, que puedan dar una gran cantidad de información respecto a un fenómeno específico y a su vez permitir establecer explicaciones generalizables, que trasciendan al contexto específico del estudio realizado (Izcara Palacios, 2007). Para alcanzar los objetivos de este estudio, se realizó un muestreo intencional, mediante la selección de individuos que permitiera realizar comparaciones significativas, atendiendo las preguntas de investigación y el tipo de explicación a desarrollar.

Dentro de las tipologías de muestreo intencional descritas por Izcara Palacios (2007) se utilizó el muestreo según determinados criterios en el cual solamente se seleccionan individuos que cumplen una serie de criterios relevantes para el diseño específico de la investigación. Se usó como fuente primaria la información documental de publicaciones periódicas especializadas de la época en que fueron construidos estos edificios, estudios recientes sobre su valor patrimonial y los documentos técnicos de soporte de la declaratoria de los Bienes Inmuebles de Interés Cultural de la ciudad de Cali (Ministerio de Cultura, 2015). Atendiendo los objetivos descritos de la investigación, se identificaron los edificios en altura, con uso de oficinas, construidos en el actual centro histórico de la ciudad de Cali, durante los inicios del Movimiento Moderno a mediados del siglo XX. Como parámetro de altura de la edificación se utilizó la caracterización contenida en Reglamento Colombiano de Construcción Sismo Resistente NSR-10 (Ministerio de Ambiente, 2010) que considera edificios de gran altura aquellos con una planta habitable a más de 15 metros sobre el nivel de la calle. Para la época, estas edificaciones eran las más altas de la ciudad.

Para garantizar que los casos de estudio tuvieran la calidad arquitectónica mínima necesaria y evitar criterios subjetivos, sólo se incluyeron en el universo de la muestra los edificios declarados Bienes Inmuebles de Interés Cultural del Ámbito Municipal con Uso Institucional (BIC) según la resolución 4132 de 2016 expedida por la alcaldía de Santiago de Cali (Concejo Municipal de Santiago de Cali, 2007). Este criterio de inclusión garantiza que las edificaciones conservan un alto porcentaje de originalidad y que no han sido sustancialmente alteradas durante el tiempo. El diseño de selección del muestreo, que se describe gráficamente en la Figura 2, permitió filtrar el universo muestral compuesto por los 83 edificios BIC incluidos en este listado, para identificar aquellos que cumplen con los criterios determinados de tiempo, uso, altura, y localización.

ACE, 16 (4.7) CC BY-ND 3.0 ES | UPC Barcelona, España | Método para la selección de casos de estudio: adaptación 
Figura 2. Proceso metodológico desarrollado

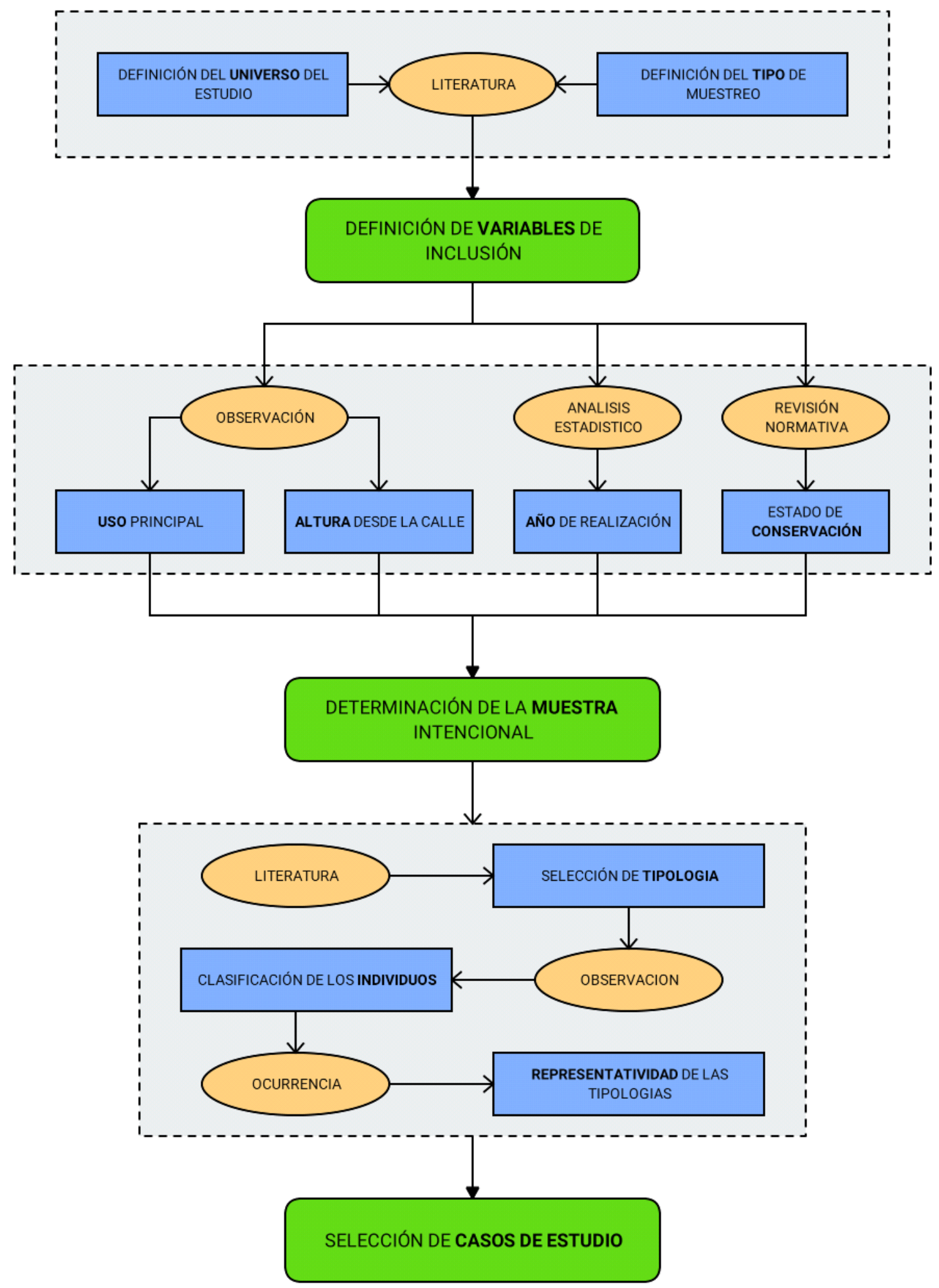

Fuente: Elaboración propia.

ACE, 16 (47) CC BY-ND 3.0 ES | UPC Barcelona, España | Método para la selección de casos de estudio: adaptación climática del Movimiento Moderno en el clima cálido ecuatorial. DOI: http://dx.doi.org/10.5821/ace.16.47.10452 
Adicionalmente, se realizó una revisión exhaustiva de la revista Proa (única publicación especializada en arquitectura de Colombia en el periodo de estudio), desde el número 1, publicado en agosto de 1946, hasta el 176, de noviembre de 1965. Para garantizar que los criterios de selección no dejaran por fuera de la muestra ningún caso representativo, se identificaron los edificios reseñados en la revista, que coinciden con Bienes Inmuebles de Interés Cultural y aquellos que deben ser excluidos de la muestra porque fueron alterados sustancialmente o demolidos para dar paso a una nueva construcción. La recopilación de información permitió depurar el universo de estudio y enfocarlo en sólo 17 casos.

El siguiente criterio de selección corresponde a la altura de la edificación. Este se consideró determinante debido a que durante el Movimiento Moderno en Colombia aparecen los primeros edificios de gran altura, que para mediados del siglo XX eran aquellos con alturas superiores a 6 pisos, atractivos gracias a la llegada de los primeros ascensores al país. Para determinar el periodo de tiempo más representativo y los valores atípicos dentro del universo de la muestra, se realizó un análisis estadístico tipo diagrama de caja y bigotes, usando la variable del año de diseño del edificio.

Mediante la información documental recopilada y las observaciones realizadas in-situ, se realizó una ficha de cada uno de los edificios estudiados y se analizaron las características geométricas y formales de los sistemas de envolvente profunda en sus fachadas. Posteriormente se agruparon por similitudes en la dirección de los elementos de sombreado predominantes y la orientación de las fachadas que los incorporan. Una vez agrupados se realizó un estudio de máscara de sombras para identificar el grado de protección solar que ofrece cada tipología según su orientación respecto a los puntos cardinales. Sobre el tamaño de la muestra se realizó un análisis porcentual para determinar los sistemas de envolvente profunda más usados en el universo estudiado y los casos atípicos.

\section{Resultados}

La selección de la muestra se inició identificando los edificios de oficinas declarados Bienes Inmuebles de Interés Cultural del Ámbito Municipal con Uso Institucional de la ciudad de Cali, Colombia. De los 82 inmuebles patrimoniales declarados BIC-M1, los 17 que aparecen en la Tabla 1 corresponden a edificios con uso de oficinas lo cual equivale al $21 \%$, que representa el mayor porcentaje, seguido por el uso educativo con 12 inmuebles como se muestra en la Figura 3. Esto demuestra la relevancia que tienen los edificios de oficinas en el patrimonio inmueble de Cali.

Al aplicar el criterio de selección correspondiente a la altura de la edificación, se determinó que de los 17 edificios de oficinas BIC-M1, 14 poseen una altura superior a 15 metros sobre el nivel de la calle, por lo cual son considerados edificios de gran altura, lo cual además concuerda con lo estipulado en la Norma Sismo Resistente NSR-10.

Para determinar el periodo más representativo se consideró que el Movimiento Moderno fue introducido en Colombia por un pequeño grupo de arquitectos e ingenieros, formados en el exterior y antes de la creación de las primeras escuelas de arquitectura del país. Las primeras edificaciones se realizaron a finales de la década de los años 40, concebidas bajo las nuevas ideas de modernidad, traídas de Europa central y Brasil y ampliamente difundidas por la revista PROA. Como universo de estudio se consideraron los 14 edificios en altura con uso de oficinas declarados BIC-M1, los cuales son los inmuebles más representativos del temprano Movimiento Moderno en Cali y aparecen sombreados en la Tabla 1. La mayoría de estos edificios están emplazados en los alrededores de las plazas de Caicedo y San Francisco y hacen parte del conjunto urbano patrimonial del centro histórico de la ciudad, cobijado por un plan de manejo y protección (Ministerio de Cultura, 2015).

ACE, 16 (4.7) CC BY-ND 3.0 ES | UPC Barcelona, España | Método para la selección de casos de estudio: adaptación climática del Movimiento Moderno en el clima cálido ecuatorial. DOI: http://dx.doi.org/10.5821/ace.16.47.10452 
La información primaria para determinar la fecha de realización de cada uno de los edificios fue obtenida de la ficha de declaratoria de los Bienes Inmuebles de Interés Cultural, que en algunos casos debió ser contrastada con los archivos fotográficos históricos. Tal es el caso del edificio del Banco de Bogotá, que aparece reseñado en 1949, pero que mediante fotografías de la época se pudo determinar que fue construido después de los edificios Sierra y Upiano Lloreda, ambos reseñados en 1950 y que conforman el costado norte de la Plaza de Caicedo. En el caso de los edificios Telecom y Banco Cafetero, que se demostró mediante fotografías que fueron construidos de manera simultánea, a pesar de que la información oficial asegura que existen 2 años de diferencia entre ellos.

Tabla 1 Listado de edificios de oficinas relacionados como Bienes de Interés Cultural del Ámbito Municipal de Cali, BIC-M1

\begin{tabular}{llllc}
\hline \multicolumn{1}{c}{ CÓDIGO } & \multicolumn{1}{c}{ NOMBRE } & AÑO & AUTOR & PISOS \\
\hline \hline BIC-M1-3 & Banco Agrario & 1930 & Borrero y Ospina & 3 \\
\hline BIC-M1-4 & Banco Cafetero & 1959 & Borrero, Zamorano y Giovannelli & 16 \\
\hline BIC-M1-5 & Banco de Colombia & 1952 & Borrero, Zamorano y Giovannelli & 11 \\
\hline BIC-M1-6 & Banco Central Hipotecario & 1965 & Samuel García, Pablo Marulanda & 12 \\
\hline BIC-M1-7 & Banco Industrial Colombiano & 1958 & Borrero, Zamorano y Giovannelli & 11 \\
\hline BIC-M1-23 & Banco de Bogotá & 1950 & Borrero y Ospina & 11 \\
\hline BIC-M1-24 & Edificio Belmonte & 1957 & Borrero, Zamorano y Giovannelli & 11 \\
\hline BIC-M1-25 & Edificio Cementos del Valle & 1955 & Borrero y Ospina & 9 \\
\hline BIC-M1-26 & Edificio Colseguros & 1950 & Borrero y Ospina & 10 \\
\hline BIC-M1-27 & Edificio Coltabaco & 1934 & Guillermo Garrido Tovar & 4 \\
\hline BIC-M1-30 & Edificio de Telecom & 1959 & Borrero y Ospina & 15 \\
\hline BIC-M1-32 & Edificio Garcés & 1940 & Moschner y Löhr & 2 \\
\hline BIC-M1-34 & Edificio Upiano Lloreda & 1950 & Borrero y Ospina & 10 \\
\hline BIC-M1-38 & Edificio Zaccour & 1952 & Philippe Mondineau & 11 \\
\hline BIC-M1-39 & Edificio Sierra & 1950 & Cuellar, Serrano y Gómez & 11 \\
\hline BIC-M1-40 & Edificio Suramericana & 1956 & Federico Blodek & 12 \\
\hline BIC-M1-81 & Centro Administrativo Municipal & 1968 & Esguerra, Sáenz y Samper & 16 \\
\hline
\end{tabular}

Fuente: elaboración propia, 2020.

Figura 3. Distribución de los edificios BIC-M1

A) Distribución por usos en los edificios

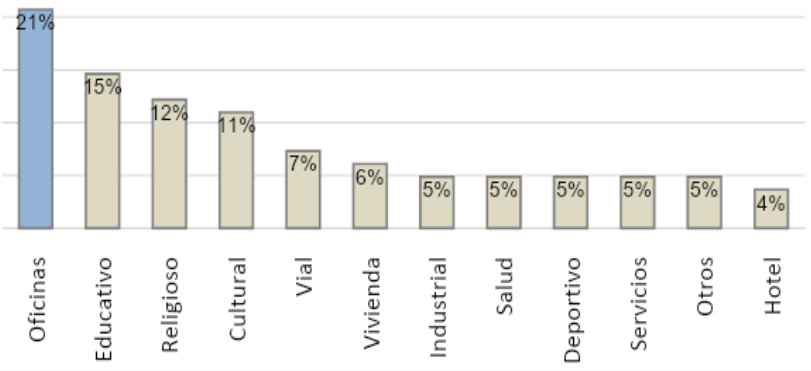

B) Distribución por año de realización

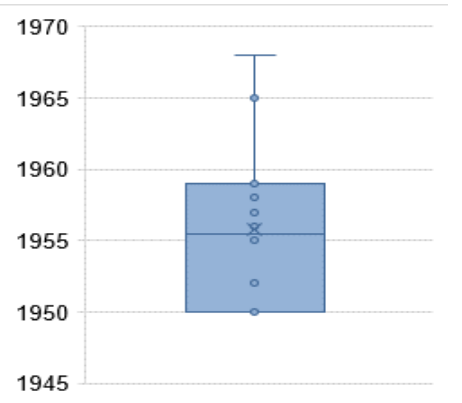

Fuente: elaboración propia, 2020 CC BY-NC-SA.

La Figura 3 muestra que el periodo más representativo para determinar los casos de estudio está entre 1950 y 1959, años en los cuales se desarrollaron el 86\% de los edificios de oficinas en altura, que representan los 12 edificios seleccionados como muestra intencionada y que se pueden apreciar en la Tabla 2.

ACE, 16 (4.7) CC BY-ND 3.0 ES | UPC Barcelona, España | Método para la selección de casos de estudio: adaptación climática del Movimiento Moderno en el clima cálido ecuatorial. DOI: http://dx.doi.org/10.5821/ace.16.47.10452 
Tabla 2 Tipología de la envolvente profunda de los edificios que hacen parte de la muestra intencionada del estudio

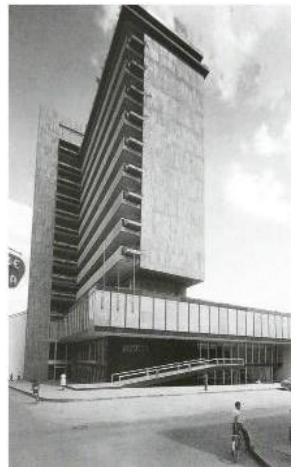

BIC-M1-4

Fachada norte con

corredor en voladizo,

fachada sur sin

dispositivos de

sombreado, fachada oeste

tipo culata

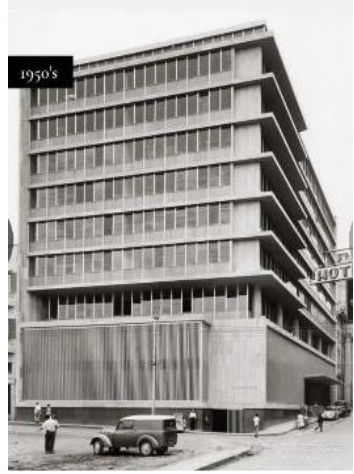

BIC-M1-5

Fachadas con elementos horizontales, más prominentes en la fachada este

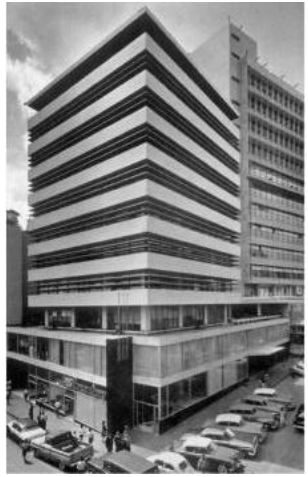

BIC-M1-7

Fachadas con igual tratamiento de

elementos horizontales hacia el norte y el este

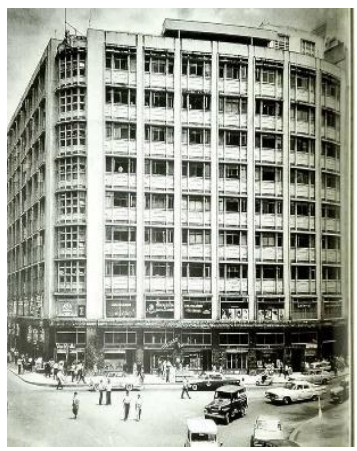

BIC-M1-26

BIC-M1-25

Fachadas norte y este con predominancia de

elementos verticales

sombreado tipo rejilla,

fachada sur sin elementos

de protección.

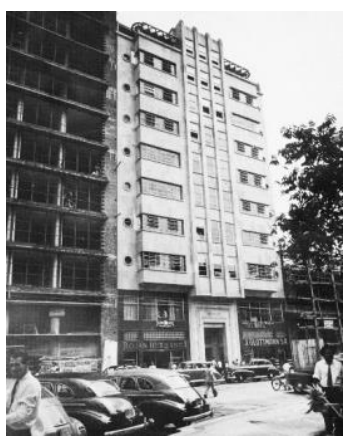

BIC-M1-34

Edificio del periodo de

transición con fachada sur que no presenta sistema de envolvente profunda

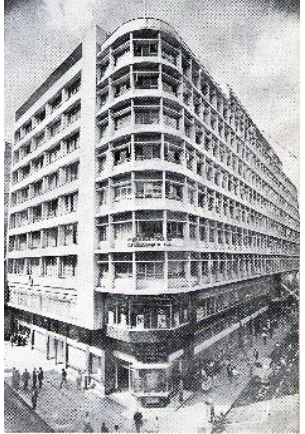

BIC-M1-38

Fachada sur con tipología combinada. Fachadas este oeste con énfasis en la horizontalidad.
Edificio del periodo de transición con fachadas sur y este que no

presenta sistema de

envolvente profunda

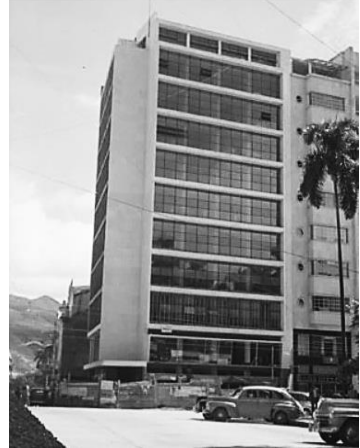

BIC-M1-39

Fachada sur con

predominancia de elementos horizontales. Fachada oeste con tipología de celosía

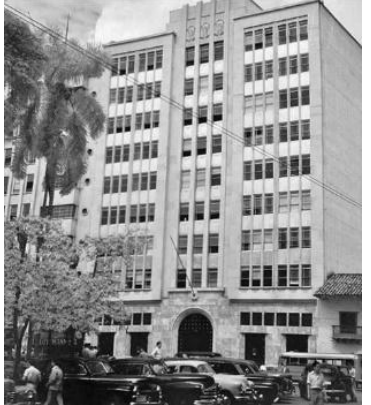

BIC-M1-23

Edificio del periodo de transición con fachada sur que no presenta sistema de envolvente profunda

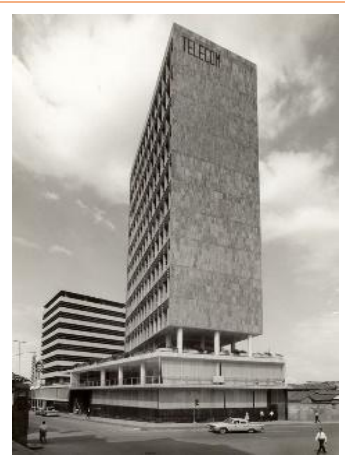

BIC-M1-27

Fachadas norte y sur con dispositivos de sombreado tipo rejilla. Fachadas este y oeste sin vanos

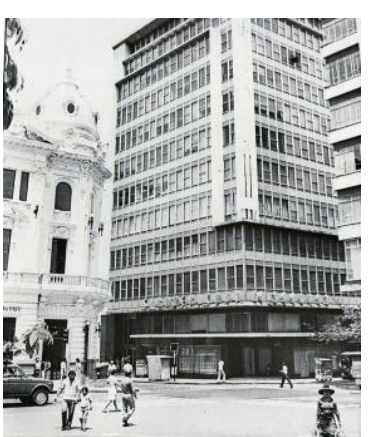

BIC-M1-40

Fachadas norte y oeste con elementos verticales poco profundos que no son considerados como envolvente profunda.

Fuente: elaboración propia, 2020.

1CE, 16 (4.7) CC BY-ND 3.0 ES | UPC Barcelona, España | Método para la selección de casos de estudio: adaptación 
Estos edificios y su localización dentro del centro histórico de Cali, se muestran en la Figura 4.

Figura 4. Localización en el centro de la ciudad de Cali de los edificios de la muestra

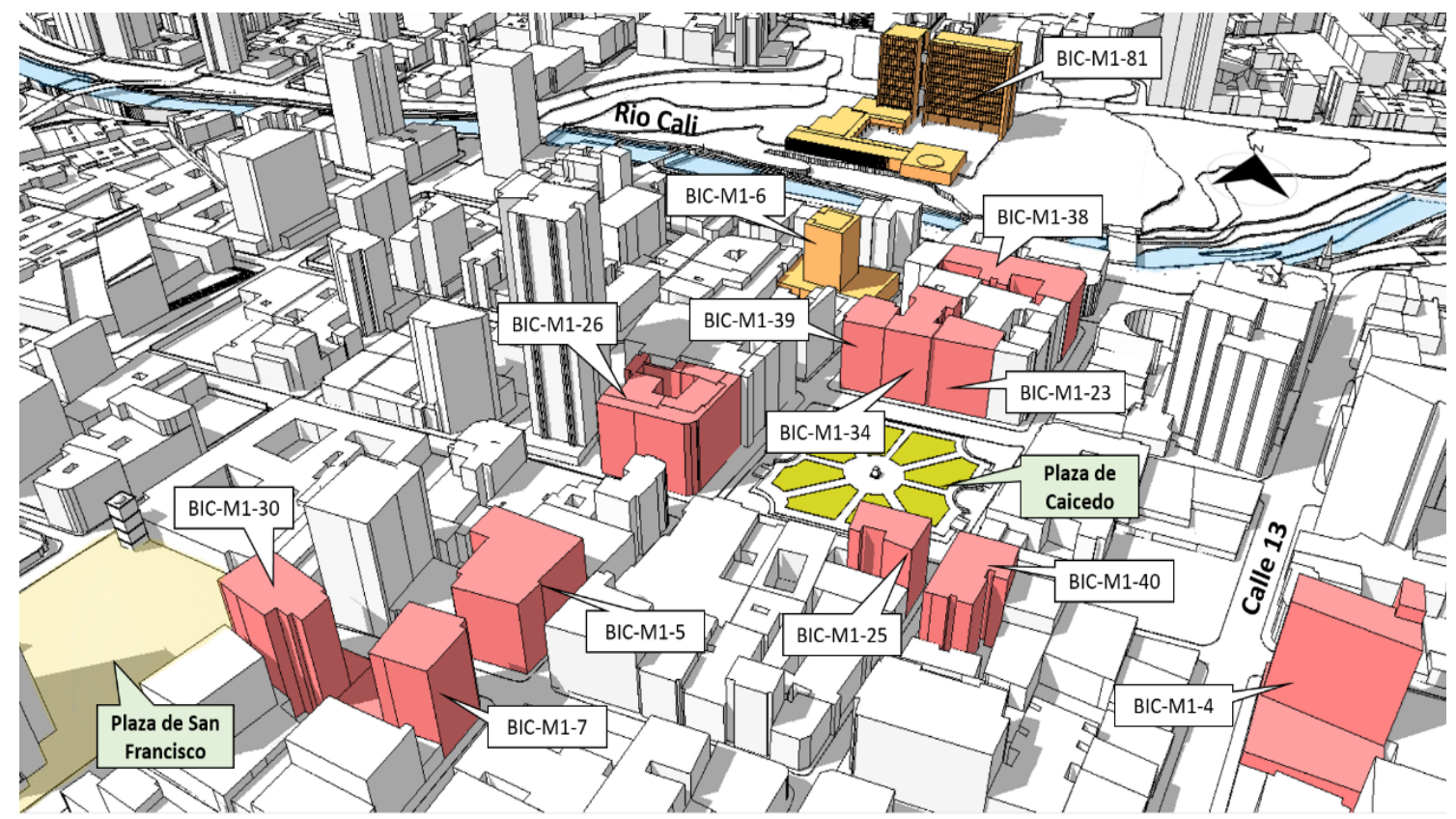

Fuente: elaboración propia, 2020 CC BY-NC-SA

En esta investigación, se consideran como envolventes profundas, los sistemas de fachadas, utilizadas en los edificios del temprano Movimiento Moderno tropical, como una estrategia para el logro del confort en los climas cálido-húmedos de la zona ecuatorial del planeta, y que, despojadas de una función de soporte estructural, generan un espacio de transición entre el interior y el exterior, con una intermediación que controla la radiación solar directa y los niveles de iluminación natural.

De los 12 edificios que hacen parte de la muestra, 3 de ellos, Colseguros (BICM1-26), Upiano Lloreda (BIC-M1-34) y Banco de Bogotá (BIC-M1-23), fueron construidos en 1950, pertenecen al período de transición, presentan un lenguaje de fachadas más cercano al Art Déco, y aún no incorporan sistemas de envolvente profunda. Así mismo, el Edificio Suramericana BIC-M1-40, posee una fachada vidriada propia del estilo internacional, con ausencia de elementos significativos de protección sombreado que generan un espacio de transición entre el interior y el exterior (Figura 5).

El único edificio de la muestra, que presenta una fachada con sistema de envolvente profunda predominantemente vertical es el Edificio de Cementos del Valle (BIC-M1-25). Son mucho más numerosos los casos encontrados de construcciones que incorporar elementos horizontales, como lo son el Banco Cafetero, el Banco Industrial Colombiano y el Banco de Colombia (Figura 6).

Entre las envolventes profundas que combinan elementos horizontales y verticales, se encuentran las celosías, presentes en las fachadas de los edificios Telecom y Belmonte, incorporadas en las fachadas largas, orientadas hacia el norte y el sur y combinadas con fachadas cortas muy cerradas hacia el este y oeste. Los edificios Sierra y Zaccour son los únicos de la muestra que combinan dos tipologías de envolvente profunda en sus fachadas. En ambos casos aparece en alguna de sus fachadas la tipología de marcos, que mezcla elementos de sombreado horizontales y verticales; y en otras fachadas elementos de sombreado predominantemente horizontales (Figura 7). 
Figura 5. Edificios del universo que no incorporar sistema de Fachada Profunda

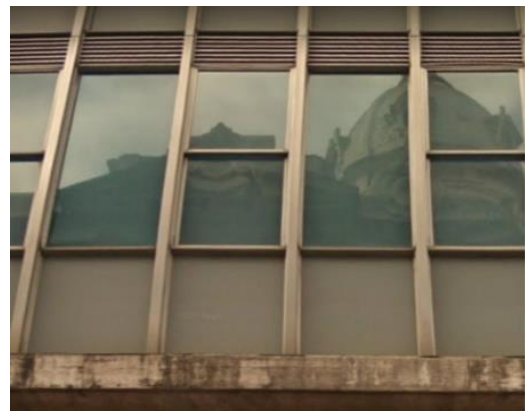

Edificio Suramericana BIC-M1-40

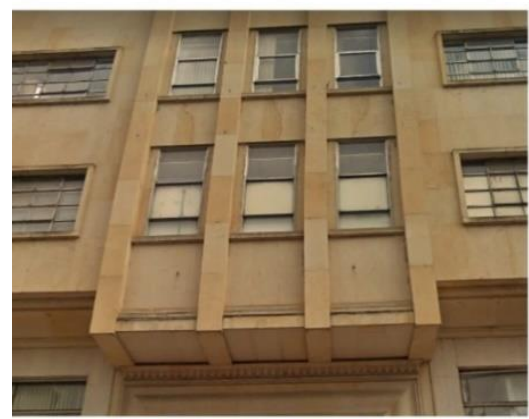

Edificio Upiano Lloreda BIC-M1-34

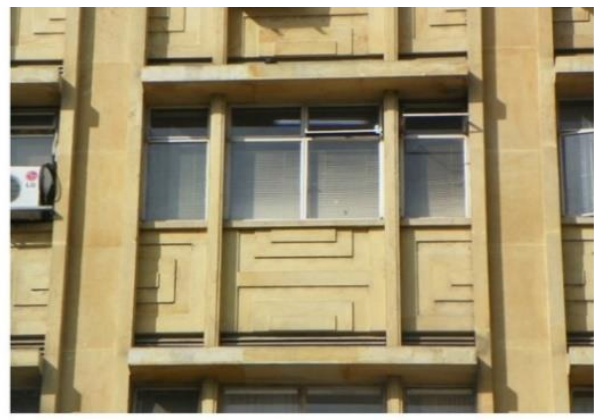

Edificio Colseguros BIC-M1-26

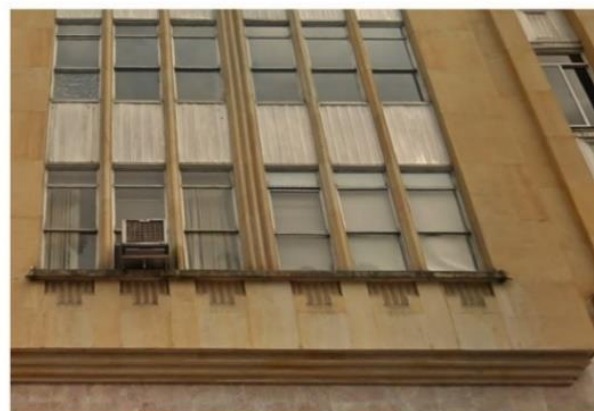

Edificio Banco de Bogotá BIC-M1-23

Fuente: elaboración propia, 2020

Figura 6. Edificios que incorporan sistemas de envolvente profunda con predominio de la verticalidad o la horizontalidad

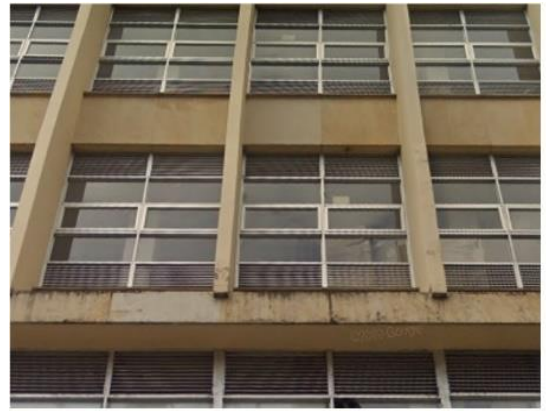

Cementos del Valle BIC-M1-25

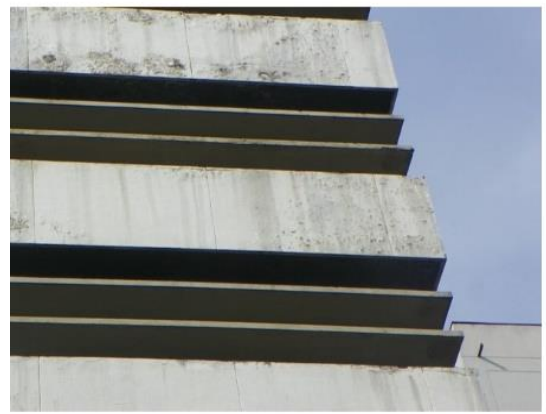

Banco Industrial Colombiano BIC-M1-7

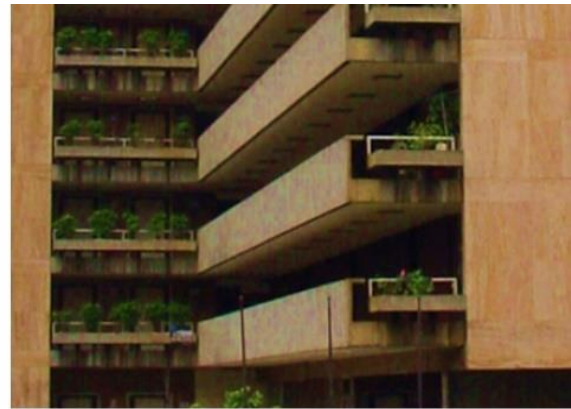

Banco Cafetero BIC-M1-4

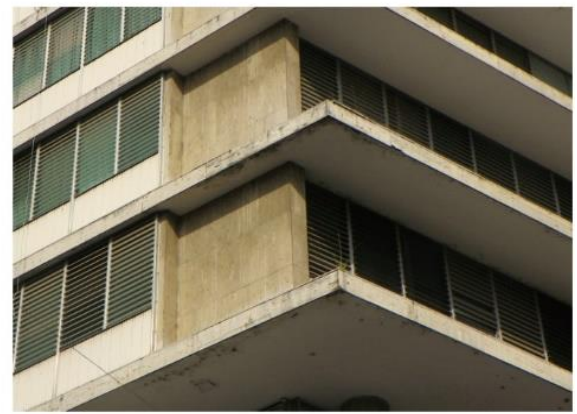

Banco de Colombia BIC-M1-5

Fuente: elaboración propia, 2020

ACE, 16 (4.7) CC BY-ND 3.0 ES | UPC Barcelona, España | Método para la selección de casos de estudio: adaptación climática del Movimiento Moderno en el clima cálido ecuatorial. DOI: http://dx.doi.org/10.5821/ace.16.47.10452 
Figura 7. Edificios que incorporan sistemas de envolvente profunda combinando elementos verticales y horizontales

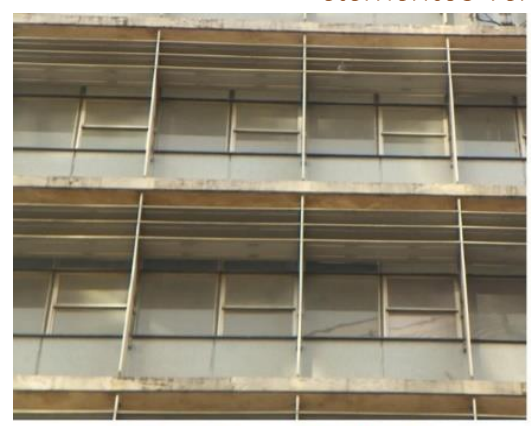

Edificio Telecom BIC-M1-30

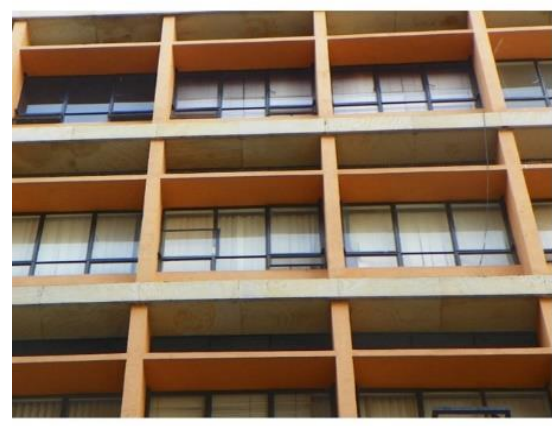

Edificio Zaccour BIC-M1-38

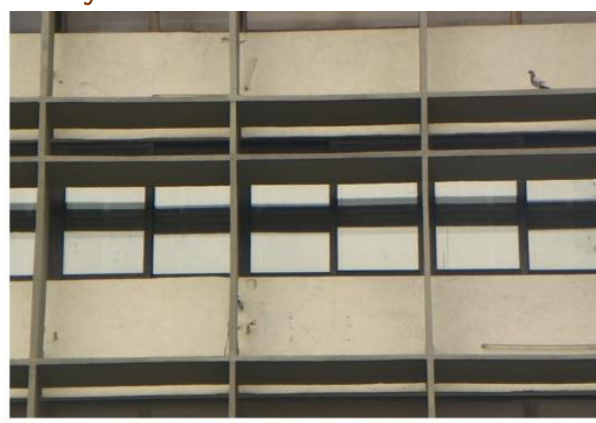

Edificio Belmonte BIC-M1-24

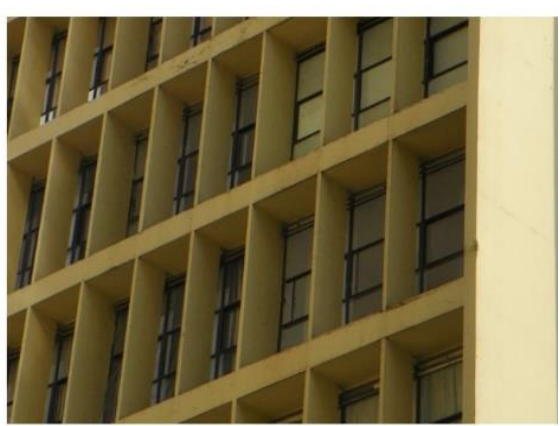

Edificio Sierra BIC-M1-39

Fuente: elaboración propia, 2020

\section{Discusión}

La investigación en arquitectura debe ser sensible a su propia naturaleza y debe ser capaz de contribuir a generar nuevos enfoques que tomen métodos desarrollados por otras disciplinas para usarlos cuando sea apropiado. La investigación cualitativa en arquitectura necesita desarrollos que den pautas respecto a los criterios de muestreo con rigor metodológico, sin tener que recurrir a las muestras tradicionales probabilísticas. El estudio de casos, que hasta hace poco era considerado en los textos de metodología como una especie de "opción blanda" con poco valor que se podía utilizar como un primer paso exploratorio de algún experimento más "riguroso", ha demostrado ser muy pertinente para la investigación en arquitectura, debido al carácter mismo de la disciplina.

Los resultados demuestran que es posible determinar las tipologías de envolvente profunda más representativas del temprano Movimiento Moderno en Cali, las cuales, de acuerdo con las condiciones climáticas del lugar pueden ser diferentes a las de otras ciudades de Colombia y de otras regiones del ecuador cálido. También se demostró la validez de la metodología utilizada para seleccionar los casos de estudio, a partir de la definición de un universo claramente determinado, que fue siendo depurado con el uso de filtros con criterios de inclusión y exclusión, hasta lograr una muestra intencionada que se adapta a las necesidades particulares de la investigación.

Se pudo determinar la relevancia que tiene la tipología de edificios de gran altura con uso de oficinas dentro del desarrollo del temprano Movimiento Moderno en Cali. De la misma forma, mediante un análisis estadístico de diagrama de cajas y bigotes se pudo ajustar el periodo de estudio, que en el

ACE, 16 (47) CC BY-ND 3.0 ES | UPC Barcelona, España | Método para la selección de casos de estudio: adaptación 
universo se había determinado entre 1945-1965, limitándolo a los años 1950-1959 en los cuales se construyeron el $86 \%$ de los edificios considerados.

Estos resultados deben ser mirados con cautela, ya que la investigación se centra en los edificios que en la actualidad conservan un alto grado de originalidad. Por lo tanto, se descartaron algunos muy buenos ejemplos de la época, que fueron reseñados en su momento por la revista Proa, pero que fueron demolidos o modificados de manera sustancial, cambiando su fachada por un lenguaje más aceptado comercialmente en la década de 1980s. Este es el caso del edificio del Banco de la República, terminado antes de 1950 y que fue probablemente el primer ejemplo de arquitectura moderna en edificios bancarios en la ciudad de Cali, tal y como lo reseña Tascón (2000).

Figura 8. Máscara de sombras para elementos verticales en Cali

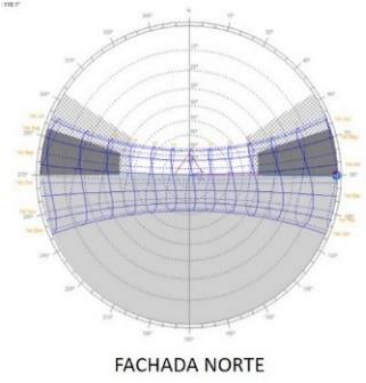

Fuente: elaboración propia

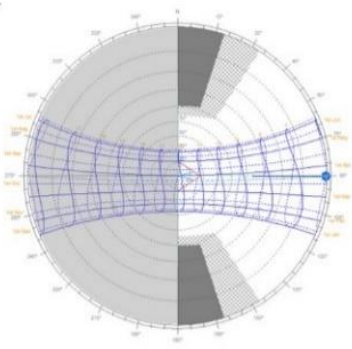

FACHADA ESTE

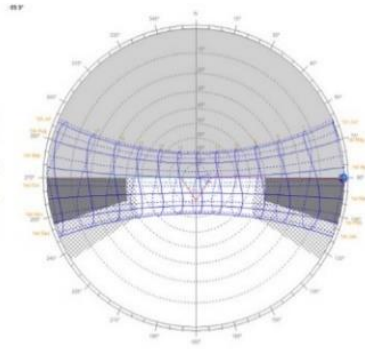

FACHADA SUR

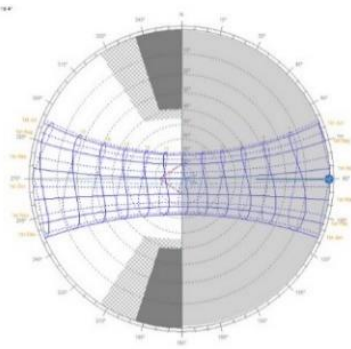

FACHADA OESTE

Llama la atención que, en el análisis realizado sobre la muestra, tan solo se encontró un edificio que utiliza un sistema de envolvente profunda, con predominio de la verticalidad, en este caso el edificio de Cementos del Valle, diseñado por la firma Borrero y Ospina, localizado en una de las esquinas de la plaza de Caicedo con 9 pisos de altura, el cual posee dos fachadas muy similares, orientadas hacia el este y el norte con quiebrasoles fijos verticales a partir del tercer piso. Un sencillo análisis de máscara de sombras, logró demostrar que esta configuración genera muy poco ocultamiento del sol y solo es efectivo en la fachada norte durante las primeras horas de la mañana y las últimas de la tarde de los meses cercanos al solsticio de junio, tal y como lo advierte Olgyay en 1968 y que se puede apreciar en la Figura 8.

Descartando los edificios Upiano Lloreda y Banco Bogotá, los dos medianeros (con frente sobre solo una vía pública), colindantes en el costado norte de la plaza de Caicedo y ambos representantes del Art déco, los demás edificios analizados se emplazan en esquinas de manzanas tradicionales de damero en el centro de la ciudad. Por esta condición todos presentan fachadas en más de una orientación.

Los edificios Telecom, Banco Cafetero y Belmonte, usan una configuración con la tipología de plataforma y torre, descrita y estudiada por Henao (2011). Sus torres se resuelven con un volumen de planta rectangular, con las caras más largas hacia el norte y el sur y las caras más cortas hacia el este y el oeste, estas últimas muy cerradas para evitar la radiación solar directa. El Banco Cafetero y Belmonte (los dos diseñados por Borrero Zamorano y Giovanelli), presentan sistemas de protección solar en sus fachadas orientadas al norte (en ambos casos dan hacia una vía pública), implementando corredores o cortasoles. Esto hace que mantengan sus fachadas orientadas al sur con grandes superficies vidriadas, completamente expuestas a la radiación solar directa, especialmente durante los meses cercanos al solsticio de diciembre. Por el contrario, el edificio Telecom, de Borrero y Ospina incorpora en sus fachadas norte y sur la misma solución de cortasoles, con combinación de elementos metálicos horizontales y verticales, logrando altos niveles de sombreado como se aprecia en la Figura 9.

ACE, 16 (4.7) CC BY-ND 3.0 ES | UPC Barcelona, España | Método para la selección de casos de estudio: adaptación 13 climática del Movimiento Moderno en el clima cálido ecuatorial. DOI: http://dx.doi.org/10.5821/ace.16.47.10452 
Figura 9. Máscara de sombras para elementos combinados en Cali

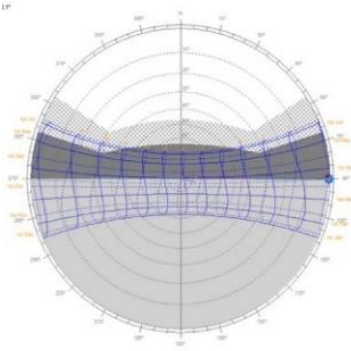

FACHADA NORTE

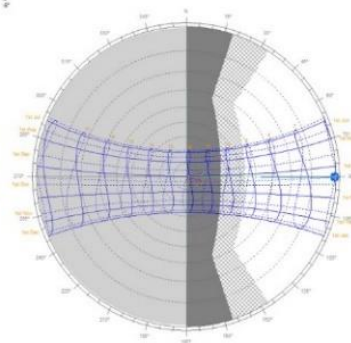

FACHADA ESTE

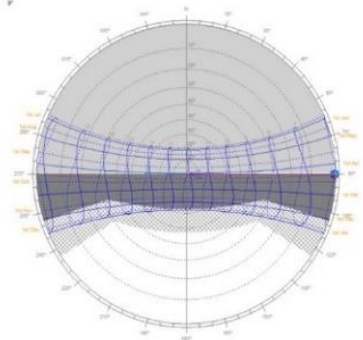

FACHADA SUR

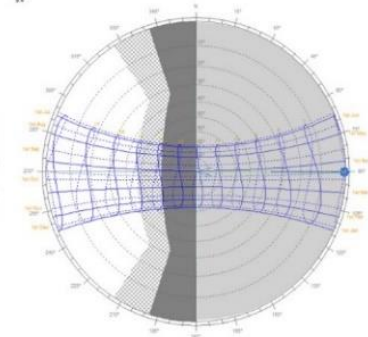

FACHADA OESTE

Fuente: elaboración propia

El edificio de suramericana presenta fachadas hacia dos calles, con orientaciones norte y oeste, ambas con grandes superficies vidriadas más cercanas al estilo internacional del edificio, con muy pocos elementos de protección solar. Los edificios del Banco de Colombia y Banco Central Hipotecario, diseñados por Borrero Zamorano y Giovanelli también utilizan la tipología de plataforma y torre, pero en estos casos las torres no son prismas de planta rectangular y poseen fachadas de tamaños similares sin prelación por la orientación hacia el norte y el sur. El Banco Industrial Colombiano incorpora fachadas idénticas hacia sus 4 lados, con elementos horizontales muy profundos que garantizan una mínima incursión solar en los momentos más críticos de cada una de las orientaciones.

Por su parte, el Banco de Colombia, presenta unas fachadas con gran porcentaje de vidrios hacia las orientaciones este y sur, protegidas con elementos horizontales del tipo alero, y que se complementan con unas pequeñas persianas horizontales que hacen parte de la ventanería. En los dos casos, la fachada profunda incorporada genera una buena protección solar como se evidencia en la Figura 10.

Figura 10. Máscara de sombras para elementos horizontales en Cali
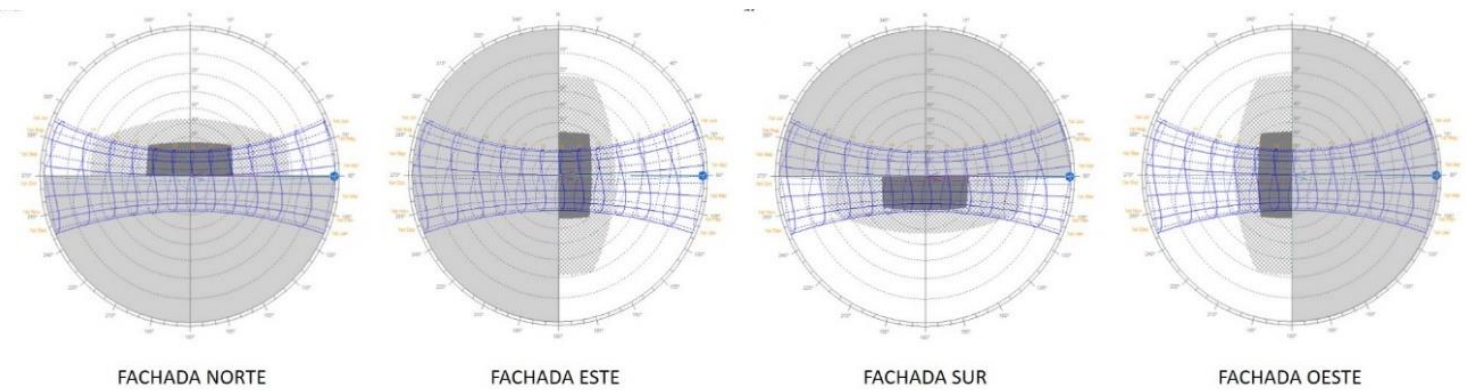

Fuente: elaboración propia

Esta revisión de los sistemas de envolvente implementados en los edificios de oficinas diseñados a mediados del siglo XX en Cali, hizo evidente que algunos casos los diseños de las fachadas parecen haber sido usados más con fines estilísticos y de imagen de modernidad, que con criterios claros de adaptación al clima. Ese es el caso de los edificios Belmonte y Banco Cafetero, que dejan las fachadas sur sin elementos de protección y concentran todos los elementos de sombreado en las fachadas norte, que dan hacia la calle, a pesar de ser esta la superficie que recibe la menor cantidad de radiación solar en la latitud específica de la ciudad.

ACE, 16 (47) CC BY-ND 3.0 ES | UPC Barcelona, España | Método para la selección de casos de estudio: adaptación climática del Movimiento Moderno en el clima cálido ecuatorial. DOI: http://dx.doi.org/10.5821/ace.16.47.10452 


\section{Conclusiones}

La revisión del estado del arte para el desarrollo de esta investigación, muestra la escasa literatura existente que oriente la toma de decisiones para la definición de muestras no probabilísticas, muy necesarias para la investigación cualitativa en arquitectura. Sin embargo, fue posible cumplir los objetivos, al poder caracterizar y clasificar los sistemas de envolvente profunda usados en los edificios del temprano Movimiento Moderno en Cali, mediante la aplicación de una metodología clara y replicable por otros investigadores interesados en trabajar con estudios de caso en arquitectura. A partir de esto, se pudieron identificar las tipologías más representativas por ser las más utilizadas y se seleccionaron los edificios que serán estudiados en detalle durante el desarrollo de un proyecto de tesis doctoral.

Los resultados de esta investigación demuestran que es posible realizar una muestra intencionada con rigor metodológico, a partir de la determinación de unos criterios específicos de selección. Se pudo demostrar la representatividad de los edificios de oficinas de gran altura, dentro del universo de los bienes inmuebles patrimoniales de la ciudad de Cali. Así mismo, fue posible determinar que el periodo 1950-1959 es el más representativo para este tipo de edificaciones, lo cual delimita el objeto de estudio de la investigación doctoral.

La decisión de utilizar una clasificación sencilla con 3 tipologías de envolvente profunda, resultó ser apropiada para el caso de Cali, a pesar de que en Colombia otros autores han optado por categorías más complejas, como es el caso de la investigación sobre los cerramientos en la arquitectura moderna en Bogotá, realizada por Montaño y Rojas (2012), quienes hacen una categorización basada en los énfasis compositivos, y proponen 5 tipologías: énfasis en la vertical, énfasis en la horizontal, énfasis combinados (vertical y horizontal), énfasis en rejillas o marcos y énfasis en patrones geométricos. Por otra parte, las investigaciones de Henao (2011) y Bustamante (2014) realizan una clasificación por climas o por su respuesta a las necesidades funcionales de la fachada. Estas investigaciones han reseñado un amplio repertorio de sistemas de envolvente profunda usados durante el Movimiento Moderno en Colombia, donde al igual que en África Central y el Sudeste Asiático, se daban las condiciones para aplicar los principios teóricos de Le Corbusier, consolidando una nueva identidad arquitectónica adaptada a las condiciones de las zonas cálidas y húmedas del planeta.

La investigación hizo evidente que en el caso de Cali, la tipología de envolvente profunda con énfasis en la verticalidad es muy poco usada, lo cual probablemente se deba a su poca eficacia en las condiciones locales, tal y como lo señala Olgyay (1968) cuando afirma que esta tipología sólo resulta eficaz para proteger de la radiación solar entre 6:00 - 9:00 am y 3:00 - 6:00 pm en las fachadas norte y sur, y que se recomienda utilizarla en combinación con elementos horizontales que protejan en las horas cercanas al medio día, que son las más calurosas.

Los edificios Zaccour y Sierra (Figura 11), localizados dentro de la misma manzana del centro histórico de Cali, se convierten en los más representativos para realizar los estudios que permitan determinar la correlación entre las características geométricas de sus envolventes profundas, y su impacto sobre las variables que determinan la Calidad del Ambiente Interior y el nivel de satisfacción de los ocupantes. Ambos casos implementan diferentes soluciones en fachada dependiendo de la orientación, y en la actualidad mantienen en gran medida los sistemas de ventilación natural originales.

Queda por determinar cuál es el desempeño de cada uno de estos sistemas de envolvente profunda en los edificios estudiados, así como su impacto en las condiciones de calidad del ambiente interior. Esto permitirá identificar las buenas prácticas en el diseño de fachadas, que se puedan durante las

ACE, 16 (47) CC BY-ND 3.0 ES | UPC Barcelona, España | Método para la selección de casos de estudio: adaptación climática del Movimiento Moderno en el clima cálido ecuatorial. DOI: http://dx.doi.org/10.5821/ace.16.47.10452 
etapas tempranas de concepción de proyectos de edificios de oficinas en el clima cálido ecuatorial. Así mismo, se abren líneas de investigación sobre la envolvente profunda como término y concepto, que estudien la importancia de estos sistemas de fachada más allá de su eficacia en la regulación de la radiación y la iluminación natural; investigando su potencial como un espacio de intermediación que diluye los límites entre el interior y el exterior.

Figura 11 Edificios Zaccour y Sierra
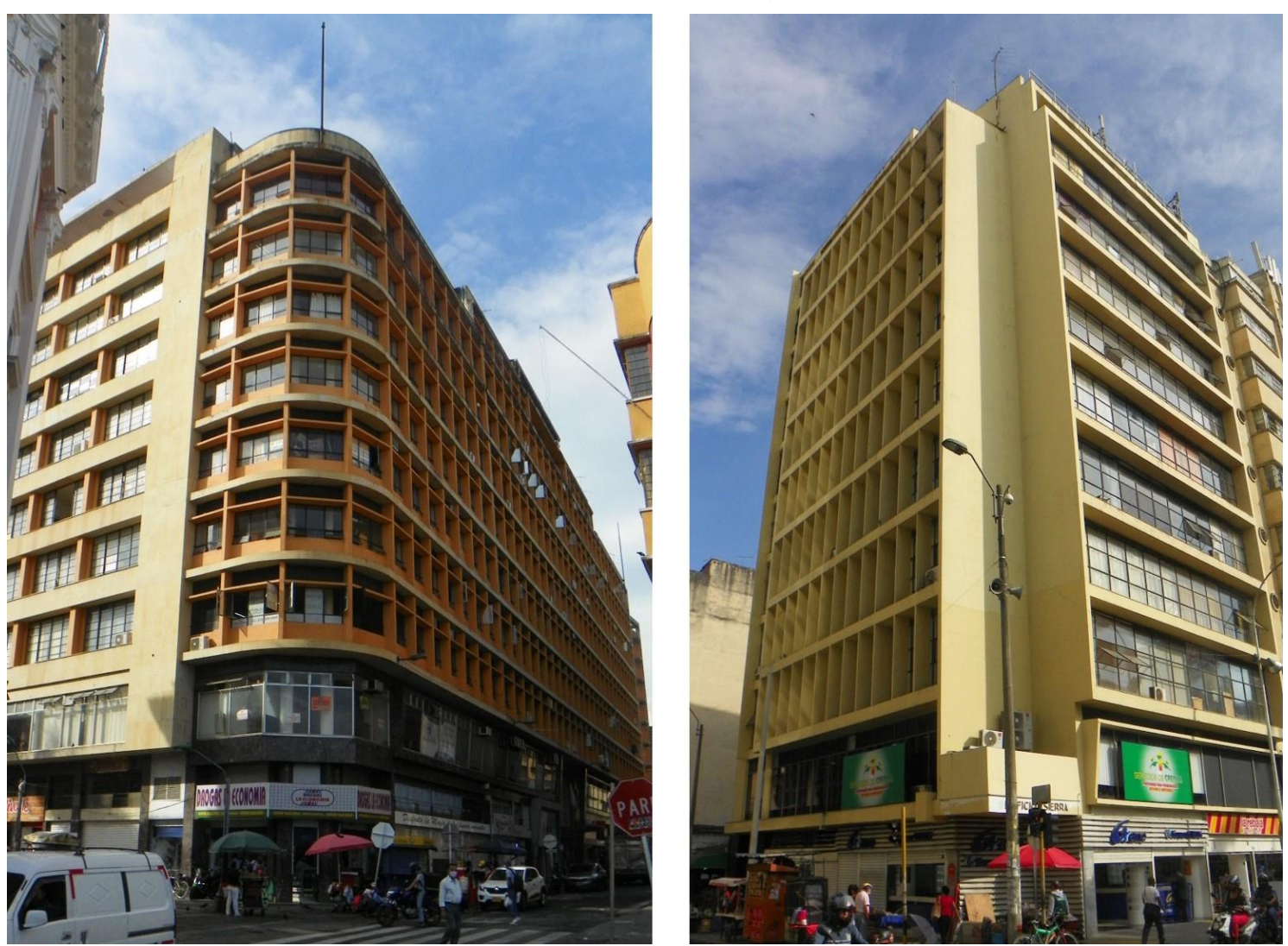

Fuente: elaboración propia

Reseñar el patrimonio moderno de Cali, es el primer paso para su conservación; y entender su desempeño energético para el logro del confort, que facilite su adaptación a los futuros estándares de eficiencia energética y edificios neto cero carbono. En este sentido, la gestión de la radiación solar es una herramienta fundamental y primaria, tal y como lo señalan Sánchez-Montañés \& Castilla (2020). Las investigaciones empíricas sobre el impacto en el confort y el consumo energético por enfriamiento de los sistemas pasivos de sombreado de fachadas, darán información útil y pertinente para la actualización de la Guía de Construcción Sostenible para el ahorro de agua y energía, que por ahora se limita a determinar un ángulo de ocultación del sol y una relación ventana pared iguales para todas las fachadas y todos los climas (Ministerio de Vivienda, 2015). Para la intervención sobre los edificios patrimoniales que pretenden atender nuevas normas de eficiencia energética, se deben considerar también criterios que eviten afectaciones sobre la envolvente y que puedan reducir su valor patrimonial (Cervero Sánchez, 2020). Se sugieren nuevas investigaciones que determinen el impacto de estos sistemas de envolvente profunda sobre las condiciones de calidad del ambiente interior que permitan identificar buenas prácticas replicables y aplicables durante las etapas tempranas de diseño. 


\section{Agradecimientos}

Al programa de formación doctoral de la Pontificia Universidad Javeriana, seccional Cali

\section{Autoría}

El primer autor realizó la conceptualización y diseño la investigación dentro de su trabajo de investigación doctoral; la segunda y tercera autoras han orientado los aspectos metodológicos y han realizado correcciones al trabajo escrito como parte de sus labores como directora y co-directora de la tesis doctoral.

Conflicto de intereses: Los autores declaran que no hay conflicto de intereses.

\section{Bibliografía}

Ali, Z. F. (2000). Environmental performance of the buildings designed by the modern masters in the tropics: architecture of Le Corbusier and Louis I. Kahn in India and Bangladesh. (Doctoral dissertation, Open University). Recuperado de https://ethos.bl.uk/OrderDetails.do?uin=uk.bl.ethos.340710

Alvarenga, A. (2013). La piel de la arquitectura moderna brasileña: las soluciones de la envolvente a la luz de los conceptos de la arquitectura bioclimática. TDX (Tesis Doctorals En Xarxa). Recuperado de https://upcommons.upc.edu/handle/2117/95058

Arifin, N. A., \& Denan, Z. (2015). An Analysis of Indoor Air Temperature and Relative Humidity in Office Room with Various External Shading Devices in Malaysia. Procedia - Social and Behavioral Sciences, 179, 290-296. DOI: https://doi.org/10.1016/j.sbspro.2015.02.432

Botti, G. (2019). Influences, identity and historiography in Colombia: the reception of Brazilian modernism (1940s-1960s). Journal of Architecture, 24(6), 731-755. DOI: https://doi.org/10.1080/13602365.2019.1684971

Bustamante Parra, D. M. (2014). La profundidad de la envolvente. Recuperado de http://www.bdigital.unal.edu.co/44367/

Carl, T. (2019). Deep Skin Architecture. In Deep Skin Architecture. DOI: https://doi.org/10.1007/978-3658-26333-1

Cervero Sánchez, N. (2020). La envolvente en la rehabilitación de conjuntos de vivienda protegida. ACE Architecture, City and Environment, 14(42), 8960. DOI: http://dx.doi.org/10.5821/ace.14.42.8960

Concejo Municipal de Santiago de Cali. (2007). Acuerdo 232 de 2007 [Consejo de Santiago de Cali] Por medio del cual se adopta el plan especial de protección del patrimonio urbano - arquitectónico del municipio de santiago de Cali. Bolentin Oficial 244,1-122. Recuperado de http://www.cali.gov.co/

CVC, DAGMA, \& CIAT. (2016). Estudio para la Microzonificación Climática para el Municipio de Santiago de Cali. 44. Recuperado de https://cgspace.cgiar.org/handle/10568/72858

Fontana, M. P., \& Mayorga, M. (2006). Colombia, arquitectura moderna. Edicions ETSAB.

ACE, 16 (47) CC BY-ND 3.0 ES | UPC Barcelona, España | Método para la selección de casos de estudio: adaptación 
Freewan, A. A. Y. (2014). Impact of external shading devices on thermal and daylighting performance of offices in hot climate regions. Solar Energy, 102, 14-30. DOI: https://doi.org/10.1016/j.solener.2014.01.009

Galindo-Díaz, J., Osuna-Motta, I., \& Marulanda-Montes, A. (2020). De componer la fachada a diseñar la envolvente El ejemplo del arquitecto Juvenal Moya en Cali. Revista de Arquitectura (Bogotá). 22(1), 94-106.DOI: https://doi.org/10.14718/RevArq.2020.2776

Ghosh, A., \& Neogi, S. (2018). Effect of fenestration geometrical factors on building energy consumption and performance evaluation of a new external solar shading device in warm and humid climatic condition. Solar Energy, 169(March 2017), 94-104. DOI: https://doi.org/10.1016/j.solener.2018.04.025

Henao, E. (2011). Torre-plataforma: Colombia años 50/60. Análisis de su adaptación arquitectónica e inserción urbana en los centros de ciudad consolidada. Tesis doctoral, ETSAB-UPC.

Izcara Palacios, S. P. (2007). Introducción al muestreo. México: Editorial Miguel Ángel Porrúa. Recuperado de http://Site. Ebrary. Com/Lib/Alltitles/DocDetail. Action.

Keitsch, M. (2012). Sustainable Design: A Brief Appraisal of its Main Concepts. Sustainable Development, 20(3), 180-188. DOI: https://doi.org/10.1002/sd.1534

Kirimtat, A., Koyunbaba, B. K., Chatzikonstantinou, I., \& Sariyildiz, S. (2016). Review of simulation modeling for shading devices in buildings. Renewable and Sustainable Energy Reviews, 53, 23-49. DOI: https://doi.org/10.1016/j.rser.2015.08.020

L'Heureux, E. (2016). Deep Envelope, Thick Elevation, and All Things Architectural on the Equator. Centre for Advanced Studies in Architecture, Department of Architecture, School of Design and Environment, National University of Singapore.

Lau, A. K. K., Salleh, E., Lim, C. H., \& Sulaiman, M. Y. (2016). Potential of shading devices and glazing configurations on cooling energy savings for high-rise office buildings in hot-humid climates: The case of Malaysia. International Journal of Sustainable Built Environment, 5(2), 387-399. DOI: https://doi.org/10.1016/j.ijsbe.2016.04.004

Lidelöw, S., Örn, T., Luciani, A., \& Rizzo, A. (2019). Energy-efficiency measures for heritage buildings: A literature review. Sustainable Cities and Society, 45(2018), 231-242. DOI: https://doi.org/10.1016/j.scs.2018.09.029

Llanos, I. (2008). Arquitectura residencial Obregón \& Valenzuela. Casa William Villa Uribe (Demolida). Dearq. Revista de Arquitectura, (2), 54-63. Recuperado de https://revistas.uniandes.edu.co/doi/pdf/10.18389/dearq2.2008.07

Lovel, J. (2013). Building envelopes: an integrated approach. Princeton Architectural Press.

Martínez-Molina, A., Tort-Ausina, I., Cho, S., \& Vivancos, J. L. (2016). Energy efficiency and thermal comfort in historic buildings: A review. Renewable and Sustainable Energy Reviews, 61, 70-85. DOI: https://doi.org/10.1016/j.rser.2016.03.018

Melendo, J. M. A., Lainez, J. M. C., \& Verdejo, J. R. J. (2008). Nineteen thirties architecture for tropical countries: Le corbusier's brise-soleil at the Ministry of Education in Rio de Janeiro. Journal of Asian Architecture and Building Engineering, 71), 9-14. DOI: https://doi.org/10.3130/jaabe.7.9

ACE, 16 (4.7) CC BY-ND 3.0 ES | UPC Barcelona, España | Método para la selección de casos de estudio: adaptación 18 climática del Movimiento Moderno en el clima cálido ecuatorial. DOI: http://dx.doi.org/10.5821/ace.16.47.10452 
Ministerio de Ambiente, V. y D. territorial. B. (2010). Reglamento Colombiano de Construcción Sismo Resistente NSR-10. Reglamento Colombiano de Construcción Sismo Resistente.

Ministerio de Cultura. Resolución 1810 de 2015 [Ministerio de Cultura] Por la cual se aprueba el Plan Especial de Manejo y Protección del Centro Histórico del municipio de Santiago de Cali y su zona de influencia, en el departamento del Valle del Cauca, declarado monumento naci. , Junio 23 § (2015).

Ministerio de Vivienda. (2015). Resolución 549 de 2015 - Anexo No. 1. (1), 1-85. Retrieved from http://www.minvivienda.gov.co/Documents/ViceministerioVivienda/ANEXO 10549 - 2015.pdf

Mirrahimi, S., Mohamed, M. F., Haw, L. C., Ibrahim, N. L. N., Yusoff, W. F. M., \& Aflaki, A. (2016). The effect of building envelope on the thermal comfort and energy saving for high-rise buildings in hot-humid climate. Renewable and Sustainable Energy Reviews, 53, 1508-1519. DOI: https://doi.org/10.1016/j.rser.2015.09.055

Montaño Bello, A., \& Rojas Farías, R. (2012). Técnica y estética en el límite moderno: los cerramientos en la arquitectura moderna en Bogotá. Dearq, (10), 88-101. DOI: https://doi.org/10.18389/dearq10.2012.10

Olgyay, V. (1968). Clima y arquitectura en Colombia. Universidad del Valle. Facultad de Arquitectura.

Osuna-Motta, I. (2017.). Enseñanza y aprendizaje en construcción sostenible: Proyecto Casa Alero. Em: XIV Encontro Nacional de Conforto no Ambiente Construído e X Encontro Latino-Americano de Conforto no Ambiente Construído. Balneário Camboriú, Brasil, 27.

Patsavos, N., \& Zavoleas, Y. (2014). SURFACE/Digital Materiality and the New Relation between Depth and Surface (Vol. 6). Recuperado de https://www.eaae.be/wpcontent/uploads/2017/04/EAAE_Transactions_48.pdf

Perdomo, L. G. (2013). Cali : entre el racionalismo de Harvard y el manierismo de. Revista Ciencias Humanas, 10 (1983). DOI: https://doi.org/10.21500/01235826.1738

Requena-Ruiz, I. (2016). Thermal comfort in twentieth-century architectural heritage: Two houses of Le Corbusier and André Wogenscky. Frontiers of Architectural Research, 5(2), 157-170. DOI: https://doi.org/10.1016/j.foar.2016.02.001

Requena Ruiz, I. (2011). Arquitectura adaptada al clima en el Movimiento moderno: Le Corbusier (19301960). (Disertación Doctoral, Universidad de Alicante).

Robson, C., \& McCartan, K. (2016). Real world research. John Wiley \& Sons.

Roesler, S., Kobi, M., \& Requena-Ruiz, I. (2018). Building a Brazilian Climate The Case of the House of Brazil in Paris (France). In The Urban Microclimate as Artifact (pp. 134-151). DOI: https://doi.org/10.1515/9783035615159-134

Rovira, T., \& Gastón, C. (2007). Arquitectura moderna en América Latina 1950-1965. Recuperado de http://upcommons.upc.edu/handle/2117/8097

Sadineni, S. B., Madala, S., \& Boehm, R. F. (2011). Passive building energy savings: A review of building envelope components. Renewable and Sustainable Energy Reviews, 15(8), 3617-3631. DOI: https://doi.org/10.1016/j.rser.2011.07.014

ACE, 16 (47) CC BY-ND 3.0 ES | UPC Barcelona, España | Método para la selección de casos de estudio: adaptación 
Sánchez-Montañés, B., \& Castilla, M. V. (2020). Fábricas de resiliencia. Una oportunidad para el patrimonio industrial: el caso de La Trinidad. ACE Architecture, City and Environment, 15(43), 9192. DOI: http://dx.doi.org/10.5821/ace.15.43.9192

Siret, D., Siret, D., \& Colloque, C. (2011). L 'illusion du brise-soleil par Le Corbusier To cite this version: HAL Id: halshs-00580040 L' illusion du brise-soleil par Le Corbusier. Recuperado de https://halshs.archives-ouvertes.fr/halshs-00580040

Sugár, V., Talamon, A., Horkai, A., \& Kita, M. (2020). Energy saving retrofit in a heritage district: The case of the Budapest. Journal of Building Engineering, 27July 2019), 1-20. DOI: https://doi.org/10.1016/j.jobe.2019.100982

Tascón, R. (2000). La arquitectura moderna en Cali: la obra de Borrero, Zamorano y Giovanelli. Fundación Civis.

Triana, M. A., De Vecchi, R., \& Lamberts, R. (2020). Building Design for Hot and Humid Climate in a Changing World. In: Enteria N., Awbi H., Santamouris M. (eds) Building in Hot and Humid Regions. Springer, Singapore. https://doi.org/10.1007/978-981-13-7519-4_3

U.S. Departament of Energy. (2012). 2011 Buildings Energy Data Book. Office of Energy Efficiency and Renewable Energy, $1-81 . \quad$ Retrieved from http://buildingsdatabook.eren.doe.gov/ChapterIntro2.aspx\%5Cnhttp://buildingsdatabook.eren.doe.gov /docs/DataBooks/2011_BEDB.pdf

Yin, R. K. (2017). Case study research and applications: Design and methods. Sage publications. 